\title{
Indicadores antropométricos en adolescentes mujeres y hombres del sur de Tamaulipas. Un estudio comparativo.
}

\author{
Anthropometric indicators in adolescent girls and boys in southern Tamaulipas. A comparative \\ study. \\ Antonio Robles Cerda ${ }^{a}$, Rodrigo León-Hernández ${ }^{b}$, Sarahí González Reyes ${ }^{c}$, Gregorio Méndez. \\ Santos $^{d}$, Gilda Gómez-Péresmitré ${ }^{e}$ y Silvia Platas Acevedo ${ }^{f}$
}

\begin{abstract}
:
Body weight is directly related to the state of health. Therefore the extremes of low weight and obesity represent problems that affect women and men at all stages of life. Therefore, the study objective was to compare nutritional status indicators (BMI and weight) among adolescent female and male students in southern Tamaulipas. A cross-sectional, descriptive and comparative study was carried out. The sample was made up of students from secondary schools in the municipalities of Ciudad Madero and Tampico, Tamaulipas. The sample was non-probabilistic with a convenience sampling with $n=344$. Weight and height were obtained to calculate BMI. Among the results it was found that the average BMI for the total of the sample (both sexes) was normal weight. Significant differences in weight, but not in BMI, were identified through the comparison test. In addition, a higher percentage trend towards low weight is observed in the BMI categories for both sexes. Therefore it is concluded that among the adolescent students of southern Tamaulipas a healthy nutritional status (normal weight) predominates, that the BMI is the same for both sexes, however male adolescents weigh more than women.
\end{abstract}

Keywords:

Adolescents, Body Mass Index, Body Weight

\section{Resumen:}

El peso corporal se relaciona directamente con el estado de salud. Por lo tanto, los extremos de bajo peso y obesidad representan problemas que afectan a mujeres y hombres en todas las etapas de la vida. Por lo anterior, el objetivo de estudio fue comparar indicadores del estado nutricio (IMC y peso) entre estudiantes adolescentes mujeres y hombres del sur de Tamaulipas. Se realizó un estudio transversal, descriptivo y comparativo. La muestra se formó por estudiantes de escuelas secundarias de los municipios de Ciudad Madero y Tampico, Tamaulipas. La muestra fue no probabilística con un muestreo por conveniencia con n=344. Se obtuvieron peso y estatura para calcular el IMC. Entre los resultados se encontró que la media de IMC para el total de la muestra (ambos sexos) se ubicó en normopeso. Mediante la prueba de comparación se identificaron diferencias significativas en el peso, más no en el IMC. Además, se observa una tendencia porcentual mayor hacia bajo peso en las categorías de IMC para ambos sexos. Por tanto, se concluye que entre los estudiantes adolescentes del sur de Tamaulipas predomina un estado nutricio saludable (peso normal), que el IMC es igual para ambos sexos, sin embargo, los adolescentes hombres pesan más que las mujeres.

\section{Palabras Clave:}

Adolescentes, Índice de Masa Corporal, Peso Corporal

\footnotetext{
a Universidad Autónoma de Tamaulipas, https://orcid.org/0000-0003-3351-4094, Email: aroblescerda@ gmail.com

b Autor de Correspondencia, Catedrático CONACYT, Universidad Autónoma de Tamaulipas, http://orcid.org/0000-0001-5780-4382, Email: rod_leonher@yahoo.com.mx

c Universidad Autónoma de Tamaulipas, https://orcid.org/0000-0003-4941-7694, Email: sarahi_gonzalez@hotmail.com

d Universidad Autónoma de Tamaulipas, https://orcid.org/0000-0002-4693-6037, Email: gregorio.msantos17@gmail.com

e Universidad Nacional Autónoma de México, https://orcid.org/0000-0003-1572-4012, Email: gildag@unam.mx

f Universidad Nacional Autónoma de México, https://orcid.org/0000-0001-9878-3653, Email: romsip@unam.mx
} 


\section{Introducción}

El concepto de obesidad ha evolucionado y se ha definido de diferentes maneras, el Instituto Nacional de Salud Pública de México define la obesidad como una enfermedad multifactorial, caracterizada por un aumento de grasa corporal, debida a la ingesta de energía excedente al gasto energético y, causante de diversas enfermedades crónicas no transmisibles ${ }^{1}$.Por su parte, la Organización Mundial de la Salud define sobrepeso y obesidad como "acumulación anormal o excesiva de grasa, que puede ser perjudicial para la salud"2.

El referente mundial para establecer desnutrición, normo peso, sobrepeso y obesidad en los adultos es el Índice de Masa Corporal (IMC), el cual se obtiene al dividir el peso en kilogramos entre la estatura en centímetros al cuadrado, el puntaje obtenido mediante esta fórmula se ha clasificado en categorías según diferentes autores, en algunas de ellas se identifica de desnutrición leve a moderada o severa, o en el caso de obesidad sucede lo mismo, desde sobrepeso hasta obesidad, sin embargo todas las categorizaciones incluyen una categoría como punto medio correspondiente al peso normal 0 normopeso ${ }^{3}$. Los criterios de IMC más comúnmente utilizados en México son los recomendados por la Organización Mundial de la Salud ${ }^{2}$, sin embargo, desde hace ya más de dos décadas existe una propuesta para categorizar el IMC de los adolescentes en México, los puntos de corte propuestos son: peso bajo (15-18.9), normal (19-22.9), sobrepeso (23-27) y obesidad (>27) .

La importancia de utilizar el IMC radica en que es uno de los indicadores más utilizados y confiables del estado nutricio, debido al bajo costo y la facilidad que implica su medición, así como su correlación con la grasa corporal. Es recomendado para población adolescente a excepción de los atletas. Cabe destacar que existen otras herramientas antropométricas que son indicadores nutricios de la masa corporal total en adolescentes, entre los que destacan el índice de peso para la talla (IPT) y el porcentaje de peso de referencia $(\% \mathrm{PR})^{5}$.

Los adolescentes representan la quinta parte del total de la población en América Latina y el Caribe $^{6}$. Debido al sobrepeso y la obesidad en este grupo de edad la salud pública se ha visto afectada ${ }^{7}$ ya que un adolescente obeso tiene una alta probabilidad de serlo en su edad adulta ${ }^{8}$.

La obesidad es un problema de salud que ha sido documentado en diferentes países del continente americano, algunos ejemplos son Argentina, que en 2014 reportó sobrepeso $26.4 \%$ y obesidad $14.1 \%{ }^{9}$. En Brasil el sobrepeso fue reportado entre el 25.7 y el $28.8 \%$, la obesidad entre el $10.4 \%$ y el $15.4 \%{ }^{10}$. En México, la prevalencia de sobrepeso y obesidad en mujeres fue de
$39.2 \%$ y en hombres de $33.5 \%$, específicamente la Ciudad de México tiene el mayor problema de sobrepeso y obesidad con un $41.6 \%$, seguida de la región sur $(38.6 \%)$ y la región norte $(38 \%)$, finalmente la zona centro $(31 \%)$. Al estar ubicado en la región norte, Tamaulipas se encuentra entre los estados que clasifican en el tercer lugar en sobrepeso y obesidad ${ }^{11}$. Concordando con lo anterior, la OMS y Organización Panamericana de la Salud (OPS) reportó que la prevalencia de sobrepeso y obesidad en México es de un $65 \%$ en la población adulta de ambos sexos, ligeramente por debajo de los Estados Unidos de Norteamérica que tiene un $68 \%{ }^{12}$.

Particularmente para población adolescente de ambos sexos del Estado de Tamaulipas se ha documentado una prevalencia combinada de sobrepeso y obesidad que paso de $38.1 \%$ en 2006 a $41.3 \%$ en 2012. Las mujeres $(42.9 \%)$ presentaron mayor prevalencia que los hombres $(39.6 \%)^{13}$.

La obesidad tiene origen multifactorial, entre ellos genético, medio ambiente, conductual, psicológico y social, que ocasiona un desequilibrio energético, con acumulación anormal de grasa, la contribución de cada uno de estos factores ha sido ampliamente estudiada, es así como OMS considera que los principales factores para desarrollar la obesidad son el estilo de vida sedentario y un aumento en la ingesta de alimento ${ }^{3}$.

Existe evidencia que indica que los factores genéticos participan hasta en un $40 \%$ de los casos de obesidad ${ }^{14}$. Uno de los acontecimientos de mayor relevancia en la genética de la obesidad, es el descubrimiento del gen denominado FTO (fat mass and obesity-associated gene), que ha mostrado una asociación estadísticamente significativa con el IMC y diabetes tipo $\mathrm{II}^{15}$.

El sobrepeso y la obesidad pueden causar trastornos físicos y/o psicológicos durante la adolescencia, además potencializa el riesgo de desarrollar enfermedades crónico-degenerativas como la diabetes mellitus, cardiopatías, osteoartritis y diferentes cánceres, entre otros $^{2}$. Además, está documentado que una dieta mal balanceada estuvo asociada a 11 millones de muertes en 2014, de ellas 9.5 millones fueron por enfermedades cardiovasculares, 913,090 por cáncer y 338,314 por diabetes mellitus ${ }^{16}$. 
Además de las consecuencias antes señaladas, es de suma importancia considerar que la obesidad genera costos del $2.8 \%$ del Producto Interno Bruto (PIB) a nivel mundial, cantidad muy parecida a lo que ocasiona el tabaquismo y los conflictos bélicos ${ }^{17}$. En México, el gasto en salud del PIB en 2016 fue de $2.9 \%$ (gubernamental) y $2.6 \%$ (privado), el gasto de bolsillo fue de $40.4 \%$ (del gasto total en salud) en el mismo año ${ }^{18}$.

Existe evidencia que invertir en la prevención de enfermedades no transmisibles es costo-efectivo, entre estas medidas se incluye las campañas masivas de información del daño a la salud del sobrepeso y obesidad, el etiquetado con el valor nutricional de los alimentos, la restricción de publicidad de alimentos y bebidas considerados como no saludables, dichas medidas representan un costo de 40.85 pesos por persona ${ }^{19}$. Por otra parte, los cálculos hechos por el Instituto Mexicano para la Competitividad (IMCO) estimaron un costo de 840 pesos por persona para las perdidas por el sobrepeso y obesidad, en México el sobrepeso y obesidad relacionado a la diabetes mellitus, absorbe entre el $73 \%$ al $87 \%$ de los gastos dedicados a la salud ${ }^{18}$. De acuerdo con la Organización para la Cooperación y el Desarrollo Económico (OCDE) en sus países miembros, se le atribuyen al sobrepeso el $71 \%$ de los gastos en tratamiento de diabetes, un $23 \%$ en las enfermedades cardiovasculares y un $9 \%$ de los tratamientos de los canceres relacionados al mismo ${ }^{20}$.

Con respecto a las diferencias de peso por sexo, la ENSANUT 2018 reportó específicamente en la población de adolescentes entre 12 y 19 años tenía sobrepeso $(23.8 \%)$ y obesidad (14.6\%), con mayor porcentaje en las mujeres $(41.1 \%)$ que en los hombres $(35.8 \%)$, además, Tamaulipas se encuentra con $16.9 \%$ de obesidad en este grupo de edad ${ }^{21}$. La prevalencia de sobrepeso y obesidad en el 2012 en mujeres fue de $35.8 \%$, por lo que aumento en el 2016 a 39.2\%, en cambio en los hombres en el 2012 fue $34.1 \%$ y para el 2016 disminuyó a $33.5 \%$.

Cabe señalar que en la literatura especializada se encuentran inconsistencias en los resultados que comparan el IMC entre hombres y mujeres estudiantes adolescentes. Estudios realizados con estudiantes de Costa Rica $^{23}$ y Colombia $^{24}$ indican que las mujeres presentan mayor IMC y peso que los hombres, a diferencia de otros estudios realizados con adolescentes de México $^{25}$ y España ${ }^{26}$ en lo que se refiere que los hombres presentan un IMC superior al de las mujeres.

Con base en las inconsistencias antes señaladas y en el problema de salud que representa la obesidad entre los adolescentes de ambos sexos, el objetivo de este estudio fue comparar indicadores del estado nutricio (IMC y peso) entre estudiantes adolescentes mujeres y hombres del sur de Tamaulipas.

\section{Materiales y Métodos}

Se realizó un estudio transversal, descriptivo y comparativo. La muestra se formó por estudiantes de primero, segundo y tercer grado de 3 escuelas secundarias (2 privadas y 1 pública) de los municipios de Ciudad Madero y Tampico-Tamaulipas. La muestra fue no probabilística con un muestreo por conveniencia con una $n=344$ ( $n=168$ mujeres y $n=176$ hombres).

Para medir el peso se utilizó una báscula de piso, marca Steren con capacidad para 150 kilogramos. La estatura se determinó mediante un estadímetro de madera.

El índice de masa corporal se determinó mediante la fórmula IMC= Peso $(\mathrm{kg}) /$ Talla $(\mathrm{m})^{2}$. Los puntos de corte se establecieron de acuerdo con la propuesta validada en México 4 (ver tabla 1).

Tabla 1. Puntos de corte IMC adolescentes mexicanos

\section{Índice de Masa Corporal}

\begin{tabular}{ll}
\hline Categoría & Puntos de corte \\
Bajo peso & $15-18.9$ \\
Normal & $19-22.9$ \\
Sobrepeso & $23-27$ \\
\hline Obesidad & Mayor a 27 \\
\hline
\end{tabular}

La recolección de datos se realizó mediante una encuesta individual que integró datos sociodemográficos, antecedentes patológicos familiares (en especial, diabetes mellitus, obesidad, cáncer), hábitos alimentarios, actividad física (tipo, duración, frecuencia). El peso y la talla fueron recolectados por estudiantes de la Licenciatura en Enfermería y estudiantes del doctorado en Ciencias de la Salud de la Universidad Autónoma de Tamaulipas campus Tampico. Se aplicó estadística descriptiva para variables sociodemográficas y se utilizó la $t$ de Student para muestras independientes para hacer la comparación por sexo.

Se solicitó carta de consentimiento informado por los padres debido a que los adolescentes eran menores de edad. Este estudio se apegó a la Ley General de Salud en Materia de Investigación, con la consideración de que es una investigación sin riesgo ${ }^{27}$.

\section{Resultados}

De los adolescentes encuestados el $51.2 \%$ pertenecían al sexo femenino, el resto eran masculinos, se encontraban en un rango de edad de 11 a 17 años $(\bar{X}=13.48$, $\mathrm{DE}=1.035)$, más de tres cuartas partes de la muestra cursaban secundaria publica $(79.1 \%)$, el 
porcentaje entre los tres grados de estudio (primero segundo y tercero) fue muy similar (tabla 2).

Tabla 2. Distribución porcentual sociodemográficos

\begin{tabular}{cll}
\hline & $\mathbf{f}$ & $\%$ \\
\hline Sexo & & \\
Femenino & 168 & 48.8 \\
Masculino & 176 & 51.2 \\
Tipo de escuela & & \\
Privada & 72 & 20.9 \\
Publica & 272 & 79.1 \\
Grado que cursan & & \\
Primero & 118 & 34.3 \\
Segundo & 104 & 30.2 \\
Tercero & 122 & 35.5 \\
\hline
\end{tabular}

Nota: $f=$ frecuencia, $\%=$ porcentaje.

Fuente: Cedula sociodemográfica de los participantes $n=344$

Se observó que la media de IMC para el total de la muestra (mujeres y hombres) se ubica en la categoría normopeso, lo mismo sucede con el peso y la talla. Cabe destacar que los valores mínimos y máximo de las variables IMC y peso, indican que entre los participantes existe tanto desnutrición severa como obesidad mórbida (tabla 3).

Tabla 3. Medidas antropométricas

\begin{tabular}{lllll}
\hline & $\overline{\boldsymbol{X}}$ & DE & Min & Max \\
\hline IMC & 20.94 & 4.22 & 9.10 & 40.79 \\
$\begin{array}{l}\text { Peso } \\
\text { (Kg) }\end{array}$ & 54.16 & 12.94 & 23 & 98 \\
$\begin{array}{l}\text { Altura } \\
\text { (mts) }\end{array}$ & 1.60 & .10 & 1.18 & 1.85 \\
\hline
\end{tabular}

Nota: $\overline{\boldsymbol{X}}=$ media, DE=desviación estándar, Min=mínimo, Max=máximo. Fuente: peso y estatura. $n=344$

Una vez que se clasificó el IMC en las categorías y se clasificó por sexo, se puede observar que, tanto para las mujeres como para los hombres, el mayor porcentaje se ubicó en peso normal (46.4\% y 35.8\% respectivamente), seguido de bajo peso (29.8 y $34.1 \%$ ). Cabe destacar que los menores porcentajes se ubicaron en obesidad para ambos sexos (tabla 4).

Tabla 4. Distribución porcentual de IMC por sexo

\begin{tabular}{|c|c|c|c|c|}
\hline & \multicolumn{2}{|c|}{ Mujeres } & \multicolumn{2}{|c|}{ Hombres } \\
\hline & $f$ & $\%$ & $f$ & $\%$ \\
\hline Bajo peso & 50 & $29.8 \%$ & 60 & $34.1 \%$ \\
\hline Pero & 78 & $46.4 \%$ & 63 & $35.8 \%$ \\
\hline \multicolumn{5}{|l|}{ Normal } \\
\hline Sobrepeso & 29 & $17.3 \%$ & 34 & $19.3 \%$ \\
\hline Obesidad & 11 & $6.5 \%$ & 19 & $10.8 \%$ \\
\hline
\end{tabular}

Para conseguir el objetivo de investigación "comparar indicadores del estado nutricio: peso e IMC, entre mujeres y hombres adolescentes estudiantes de Tampico Tamaulipas" se aplicó una prueba t de Student para muestras independientes. Los resultados muestran que hay diferencias significativas en el peso más, no en el IMC. Las medias en peso indican que los estudiantes adolescentes hombres presentan mayor peso en comparación con las mujeres (tabla 5).

Tabla 5. Comparación IMC y peso por sexo.

\begin{tabular}{llllllll}
\hline Variables & & \multicolumn{3}{c}{ Mujeres } & \multicolumn{2}{l}{ Hombres } \\
\hline & $\mathrm{t}$ & $\mathrm{gl}$ & Sig & Media & $\begin{array}{l}\text { Desviación } \\
\text { Estándar }\end{array}$ & Media & $\begin{array}{l}\text { Desviación } \\
\text { Estándar }\end{array}$ \\
& & & & & & & \\
Peso & -2.7 & 116 & .005 & 55.61 & 9 & 61.47 & 13.74 \\
IMC & .568 & 120 & .577 & 21.67 & 3.63 & 21.26 & 4.34 \\
\hline
\end{tabular}

Nota: $\mathbf{t}=\mathbf{t}$ de Student, $\mathbf{g l}=$ grados de libertad, Sig = significancia

Fuente: peso $n$ y
$n=344$

\section{Discusión y Conclusiones}

La muestra de estudio presentó porcentajes similares en su distribución por sexo y por grado de estudio, sin embargo, el porcentaje de pertenencia a escuela pública fue mayor al de escuela privada, a pesar de que el presente estudio se implementó en dos escuelas privadas y una pública. Lo anterior se explica ya que es común que en México las escuelas públicas tengan mayor matrícula que las escuelas públicas, lo anterior podría estar relacionada con la economía de la población, tómese en cuenta que somos un país en el que la población de bajos recursos predomina.

Con referencia a los indicadores antropométricos peso, talla e IMC, resulta favorable que tanto los puntajes promedio para la muestra total, como las categorías de IMC para mujeres y hombres, indican que predomina el peso normal entre los estudiantes, dato que concuerda con las encuestas nacionales ${ }^{13,21,22}$. Sin embargo uno de los hallazgos de interés fue que tanto para hombres como 
para mujeres se observa una tendencia porcentual mayor hacia bajo peso en las categorías de IMC, dato que difiere de los estudios estatales ${ }^{13}$, nacionales ${ }^{11,13}$, e internacionales ${ }^{9,10,12}$, donde se observa que la tendencia hacia el sobrepeso y la obesidad es mayor, representa un alto costo ${ }^{17,18}$ y representa mayor riesgo desarrollar enfermedades crónico-degenerativas.

La tendencia hacia el bajo peso entre los adolescentes de la muestra podría encontrar respuesta en las particularidades de la zona geográfica (la zona costera con mayor humedad en la República) en la que la dieta está integrada, en su mayoría, a base en pescados y mariscos. Otra posible explicación puede atribuirse a que el muestreo fue por conveniencia, por tanto, la muestra puede estar sesgada y muy probablemente no fue representativa de la población. Es decir, se estudió una muestra con características distintas al comportamiento de ese grupo etario, fenómeno que ha sido documentado en otro estudio con una muestra similar del extremo sureste de México ${ }^{28}$.

Uno de los resultados relevantes fue que no se detectaron diferencias de IMC en la comparación por sexo, sin embargo, la diferencia con el indicador "Peso" mostró que el grupo de hombres presenta un promedio de peso mayor que el grupo de mujeres. Estos resultados concuerdan con estudios antecedentes de adolescentes españoles ${ }^{26}$ y mexicanos $^{25}$, pero difiere de otros estudios de Colombia ${ }^{24}$, Costa Rica ${ }^{23}$ y México ${ }^{21}$. Por tanto, se confirma que existen inconsistencias que pueden atribuirse a las características de las muestras de estudio. Por tanto, se sugiere realizar estudios poblacionales o con muestras probabilísticas de la misma región (Sur de Tamaulipas), para confirmar que este comportamiento de los indicadores de peso es constante.

De lo anterior se concluye que los estudiantes adolescentes del Sur de Tamaulipas (CD. Madero y Tampico), en su mayoría presentan un estado nutricio saludable (peso normal), sin embargo, se documentó una tendencia hacia el bajo peso, dato que no concuerda con las encuestas nacionales. Con respecto a la comparación por sexo en los indicadores antropométricos, se concluye que el IMC es igual para ambos, sin embargo, los adolescentes hombres pesan más que las mujeres de esta Zona.

\section{Agradecimientos}

Agradecemos al proyecto titulado Prevención Primaria. Factores de Riesgo para los problemas de peso corporal en preadolescentes. PAPIIT-IN307517

\section{Referencias}

[1] Gutiérrez JP, Rivera-Dommarco J, Shamah-Levy T, VillalpandoHernández S, Franco A, Cuevas-Nasu L, Romero-Martínez M, Hernández-Ávila M. Encuesta Nacional de Salud y Nutrición 2012. Resultados Nacionales. Cuernavaca, México: Instituto Nacional de Salud Pública (MX), 2012.

[2] Organización Mundial de la Salud. Obesidad y sobrepeso [monografía en Internet]. OMS, 2018. [accesado 10 de febrero 2020]. Disponible en: https://www.who.int/es/news-room/fact-sheets/detail/obesity-andoverweight

[3] World Health Organization. Obesity: preventing and managing the global epidemic. World Health Organization, 2000.

[4] Gómez PG, Saucedo MT. Validez diagnostica del IMC (índice de masa corporal) en una muestra de escolares preadolescentes y adolescentes mexicanos. Acta Pediatr Méx. 1997;18(3):103-110.

[5] Ravasco P, Anderson H, Mardones F. Métodos de valoración del estado nutricional. Nutrición Hospitalaria. 2010;25(3):57-66

[6] Unicef. "Una aproximación a la situación de adolescentes y jóvenes en América Latina y el Caribe"; 2015. Recuperado de https://www.unicef.org/lac/sites/unicef.org.lac/files/201804/UNICEF_Situacion_de_Adolescentes_y_Jovenes_en_LAC_junio 2105.pdf

[7] Organización Mundial de la Salud. Informe de la Comisión para acabar con la obesidad infantil. Ginebra: OMS; 2016.

[8] Gordon-Larsen P, Adair LS, Nelson MC, Popkin BM. Five-year obesity incidence in the transition period between adolescence and adulthood: the National Longitudinal Study of Adolescent Health. The American Journal of Clinical Nutrition.2004;80(3):569-575. https://doi.org/10.1093/ajen/80.3.569

[9] Catalani F, Fraire J, Pérez N, Mazzola M, Martínez AM, Mayer MA. Prevalencia de bajo peso, sobrepeso y obesidad en adolescentes escolarizados de la provincia de La Pampa. Arch Argent Pediatr. 2016;114(2):154-158. http://dx.doi.org/10.5546/aap.2016.154

[10] Niehues JR, González AI, Lemos RR, Bezerra PP, Haas P. Prevalence of overweight and obesity in children and adolescents from the age range of 2 to 19 years old in Brazil. Int J Pediatr.2014;2014:583207.

[11] Shamah-Levy T, Cuevas-Nasu L, Gaona-Pineda EB, Gómez-Acosta LM, Morales-Ruán MC, Hernández-Ávila M, Rivera-Dommarco JA. Sobrepeso y obesidad en niños y adolescentes en México, actualización de la Encuesta Nacional de Salud y Nutrición de Medio Camino 2016. Salud Pública Mex. 2018; 60:244-253. https://doi.org/10.21149/8815

[12] Organización Mundial de la Salud. Estrategia de cooperación de la organización panamericana de la salud/organización mundial de la salud con México 2015-2018. 2015. https://www.paho.org/mex/index.php?option=com_docman\&view=do wnload\&category_slug=technical-documentation\&alias=1053-ccsmex-1518-web0\&Itemid=493

[13] Instituto Nacional de Salud Pública. Encuesta Nacional de Salud y Nutrición 2012. Resultados por entidad federativa, Tamaulipas. Cuernavaca, México: Instituto Nacional de Salud Pública, 2013. Disponible en: encuestas.insp.mx

[14] Loos RJ, Bouchard C. Obesity - Is it a genetic disorder? Journal of Internal Medicine. 2003;254: 401-425. https://doi.org/10.1046/j.13652796.2003.01242.x

[15] Loos RJ, Bouchard. C, FTO: The first gene contributing to common forms of human obesity. Obesity Reviews.2008;9(3):246-250. https://doi.org/10.1111/j.1467-789X.2008.00481.X

[16] Afshin A, Sur PJ, Fay KA, Cornaby L, Ferrara G, Salama JS, et al. Health effects of dietary risks in 195 countries, 1990-2017: a systematic analysis for the Global Burden of Disease Study 2017. The Lancet. 2019;393(10184):1958-1972. https://doi.org/10.1016/S01406736(19)30041-8 
[17] Dobbs R, et al. Overcoming obesity: An initial economic analysis. McKinsey Global Institute, 2014. 2016.[18] Instituto Mexicano para la Competitividad. Kilos de más, pesos de menos: los costos de la obesidad en México. 2015. http://imco.org.mx/wpcontent/uploads/2015/01/20150127_ObesidadEnMexico_Documento Completo.pdf

[19] Cecchini M, Sassi F, Lauer JA, Lee YY, Guajardo-Barron V, Chisholm D. Tackling of unhealthy diets, physical inactivity, and obesity: Health effects and cost-effectiveness. The Lancet. 2010;376(9754):1775-84: https://doi.org/10.1016/S0140-6736(10)61514-0

[20] OCDE. Heavy Burden of Obesity: The Economics of Prevention. Paris. OECD Publishing. 2019

[21] Encuesta Nacional de Salud y Nutrición 2018. Presentación de resultados presentado en; 2019; Ciudad de México.

[22] Secretaria de Salud. Encuesta Nacional de Salud y Nutrición de Medio Camino 2016. 2016.

[23] Barquero-Jiménez D, Zúñiga-Marín J, Villanueva-Castro B, BarrantesFernández M, Valverde-Arias K. Índice de masa corporal (IMC) y su relación con la edad en una población de estudiantes de secundaria en el Colegio de San Pedro de Poás. Costa Rica. 2017. https://rstudiopubs-

static.s3.amazonaws.com/328342_1f638ec407204e1c9f2c11a6ddbac8 bd.html

[24] Ortega-Bonilla R, Chito-Trujillo D. Valoración del estado nutricional de la población escolar del municipio de Argelia, Colombia. Revista de salud pública. 2014;16(4):547-559. doi: 10.15446 / rsap.v16n4.40658

[25] Del campo CJ, González, GL; Gámez RA. Relación entre el índice de masa corporal, el porcentaje de grasa y la circunferencia de cintura en universitarios. Investigación y Ciencia, 2015; 23(65):26-32. https://www.redalyc.org/pdf/674/67443217004.pdf

[26] Ramos P, Francisco R, Soledad PR, Lara L, Moreno C. Diferencias de género en la imagen corporal y su importancia en el control de peso. Escritos de Psicología, 2016;9(1):42-50 http://scielo.isciii.es/pdf/ep/v9n1/informe5.pdf

[27] Secretaria de Salud. Reglamento de la ley general de salud en materia de investigación para la salud, 2014. Recuperado de http://www.salud.gob.mx/unidades/cdi/nom/compi/rlgsmis.html

[28] Oliva-Peña Y, Ordóñez-Luna M, Santana-Carvajal A, Marín-Cárdenas A, Andueza PG, Gómez CI. Concordancia del IMC y la percepción de la imagen corporal en adolescentes de una localidad suburbana de Yucatán. Revista biomédica, 2016;27(2):49-60. http://dx.doi.org/10.32776/revbiomed.v27i2.24 